\title{
Microbial biotransformation of two phosphonoacetic acid derivatives bearing two stereomeric centres
}

\author{
Jagoda Szyszkowiak, Paulina MaJewska* \\ Department of Bioorganic Chemistry, Faculty of Chemistry, Wrocław University of Technology, Wrocław, Poland \\ *Corresponding author: paulina.majewska@pwr.wroc.pl
}

\begin{abstract}
Three strains of microorganisms: Bacillus subtilis, Serratia liquefaciens and Escherichia coli were tested as whole-cell biocatalysts for the kinetic resolution of isomers of two new phosphonoacetic acid derivatives. Used compounds possess two chiral centres - one at the carbon adjacent to both functional groups and the other at the phosphorus. Biocatalytic hydrolysis of 2-butyryloxy-2-(butoxyetoxyphosphinyl)acetic acid and 2-butyryloxy-2-(isobutoxyetoxyphosphinyl)acetic acid with whole cells of Bacillus subtilis produced corresponding hydroxyphosphonates with diastereoselectivity ranging from 50 to $60 \%$.
\end{abstract}

Key words: hydroxyphosphonates, lipolytic activity, biotransformations

\section{Introduction}

In today's chemistry, biological means such as whole microbial cells or purified enzymes are increasingly being used for completing complex synthetic processes. One of the challenging applications of biocatalysis is the separation of enantiomers. Due to the fact that biological activity of many compounds is often inseparably connected with their stereochemistry, it becomes essential that pure enantiomers can be obtained at a certain point of the synthesis. Kinetic resolution of enantiomers with the use of microorganisms or enzymes is one of the routes to make that separation relatively easy and cost effective.

Hydroxyphosphonates possessing stereogenic center(s) constitute a group of substrates used for synthesis of a vast number of structurally variable organophosphonic compounds (Kolodiazhnyi, 2005; Plażuk et al., 2006; Pawar et al., 2006; Nesterov and Kolodiazhnyi, 2006; Kolodiazhnyi, 2012). Even though their properties and potential uses are yet to be discovered, they have been demonstrated to be efficient antiviral (Kolodiazhnyi, 2005; Magee and Evans, 2012), antibacterial and anticancer agents (Kolodiazhnyi, 2005). In addition, $\alpha$-hydroxyphosphonate derivatives of tetrazolo[1,5-a]quinoline were found to possess antibacterial and antifungal properties (Kategaonkar et al., 2010). Our earlier studies, aimed at obtaining pure enantiomers of organophosphonic compounds, demonstrated that some hydroxyphosphonates might be delivered with good or excellent enantioselec- tivity by means of biotransformations (Skwarczyński et al., 1999; Malinowska et al., 2011). It was also found that analogous compounds like hydroxyphosphinates, may be produced in a similar way (Majewska et al., 2006; Majewska et al., 2009).

The purpose of this study was to extend the scope of our previous research aimed at the resolution of racemic mixtures of organophosphonic compounds that may possess some potential biological activity. We focused on the hydrolysis of 2-butyryloxy-2-(butoxyethoxyphosphinyl)acetic and 2-butyryloxy-2-(isobutoxyethoxyphosphinyl)acetic acids (Fig. 1; compounds 3a and 3b) using whole-cell biocatalysis in order to obtain enantiomerically pure forms of 2-hydroxy-2-(butoxyethoxyphosphinyl)acetic acid and 2-hydroxy-2-(isobutoxyethoxyphosphinyl)acetic acid (Fig. 2; compounds 2a and 2b). Three species of bacteria, BacilIus subtilis, Escherichia coli and Serratia liquefaciens, were tested. These microorganisms were selected from several bacterial strains stored in the Department of Bioorganic Chemistry, Wrocław University of Technology collection. The selection was made on the basis of best lipolytic activity measured by Spirit blue agar test and previously observed results for the hydrolysis of butyryloxyphosphonates (Majewska et al., 2006; Malinowska et al., 2011). Compounds $\mathbf{2 a}$ and $\mathbf{2 b}$ were also chemically synthesized as a racemic mixture of all isomers, to confirm the identity of the hydrolysis products. 
<smiles>CCCCOP(=O)(OCC)C(OC(=O)CCC)C(=O)O</smiles>

$3 a$<smiles>CCCC(=O)OC(C(=O)O)P(=O)(OCC)OCC(C)C</smiles>

$3 b$

Fig. 1. Compounds $\mathbf{3 a}$ and $\mathbf{3 b}$

A

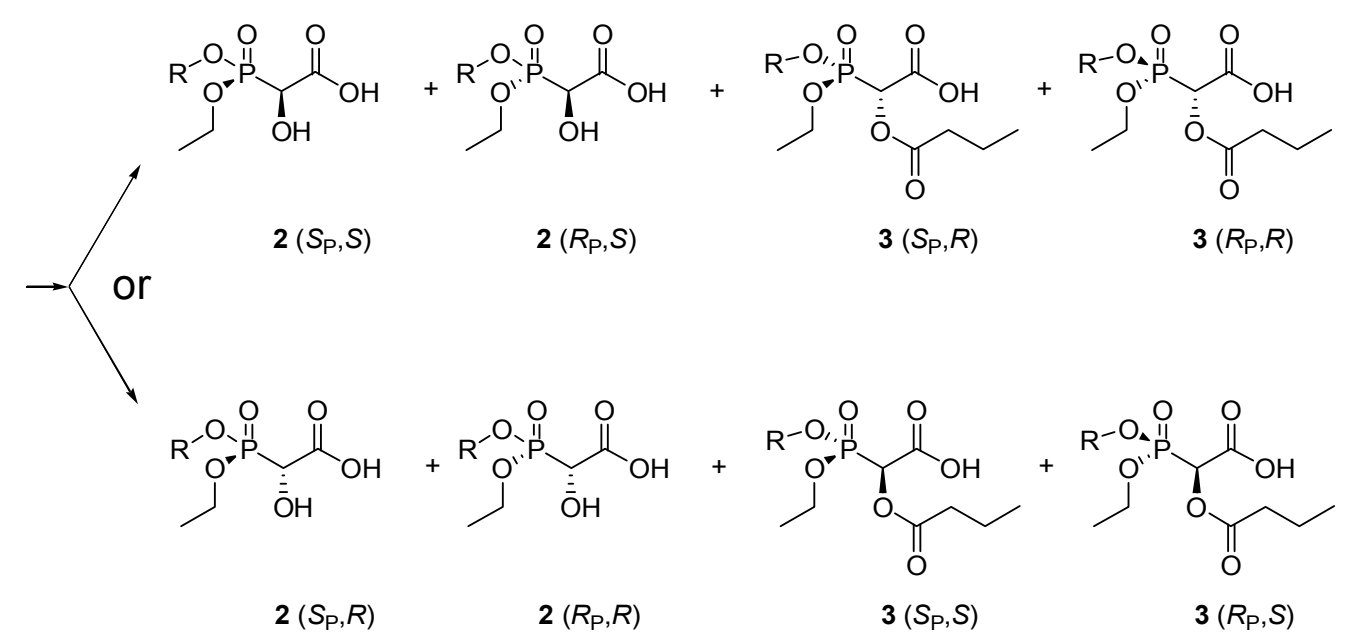

B<smiles>[R]OP(=O)(OCC)C(OC(=O)CCSC(C)C)C(=O)O</smiles>

There is no literature data suggesting that these compounds have been synthesized and explored previously. Several papers report microbial transformation of similar compounds. Kinetic resolution of enantiomers of ethyl hydroxy(phenyl)methane( $P$-phenyl) phosphinate (a compound with two stereogenic centres but without the carboxylic group) has been tested earlier, using several bacterial strains (Majewska et al., 2006). Enantioselective biooxidation by fungi of ethyl hydroxy(phenyl)- methane( $P$-phenyl) phosphinate has also been previously investigated (Klimek-Ochab et al., 2008).

\section{Materials and methods}

All materials were purchased from Sigma Aldrich (St. Louis, Missouri, United Staes), POCh (Gliwice, Poland) or BIOCORP (Warszawa, Poland) and were used without further purification. 
B. subtilis and S. liquefaciens were obtained from our own collection and were identified by the Deutsche Sammlung für Mikroorganismen und Zellkulturen (DSMZ), Braunschweig, Germany. E. coli was purchased from DSMZ.

NMR (Nucleic Magnetic Resonance) spectra were measured on a Bruker Avance ${ }^{\mathrm{TM}} 600$ at $600.58 \mathrm{MHz}$ for ${ }^{1} \mathrm{H} ; 243.12 \mathrm{MHz}$ for ${ }^{31} \mathrm{P}$ and $151.02 \mathrm{MHz}$ for ${ }^{13} \mathrm{C}_{\text {in }} \mathrm{CDCl}_{3}$ (99.8\% of atom D, contains $0.03 \% \mathrm{v} / \mathrm{v}$ TMS) or on a Bruker Avance ${ }^{\mathrm{TM}}$ DRX 300 instrument operating at 300.13 MHz for ${ }^{1} \mathrm{H} ; 121.50 \mathrm{MHz}$ for ${ }^{31} \mathrm{P}$ and $75.45 \mathrm{MHz}$ for ${ }^{13} \mathrm{C}$ in $\mathrm{CDCl}_{3}(99.8 \%$ of atom D, contains $0.03 \% \mathrm{v} / \mathrm{v}$ TMS). Chemical shifts $(\delta)$ are reported in ppm and coupling constants $(J)$ are given in Hz. $1 \mathrm{H} \mathrm{NMR}$ are referenced to the internal standard TMS $(\delta=0.00)$ and ${ }^{13} \mathrm{C}$ NMR spectra to the central line of $\mathrm{CHCl}_{3}(\delta=77.23)$.

The synthesized compounds were purified by gradient column chromatography using Merck Silica Gel 60 (63-230 mesh).

\section{Synthesis of compounds 1}

Compounds $\mathbf{1 a}$ and $\mathbf{1 b}$ were synthesized according to the method described below (Kosolapoff, 1951).

A mixture of $82.8 \mathrm{~g}(0.6 \mathrm{~mol})$ of diethyl phosphite and $44.4 \mathrm{~g}(0.6 \mathrm{~mol})$ of $n$-butanol or iso-butanol was slowly heated in a distillation apparatus (under normal pressure) up to oil bath temperature of $150^{\circ} \mathrm{C}$, when a brisk reaction is initiated and ethanol distillation began. The bath was kept at $150-155^{\circ}$ until $16 \mathrm{ml}$ of ethanol was collected. The mixture was then cooled to room temperature (RT). Next, the mixture was distilled under reduced pressure, and three fractions were collected (first at $90-100^{\circ} \mathrm{C}$, second at $100-120^{\circ} \mathrm{C}$ and third at $\left.125-140^{\circ} \mathrm{C}\right)$. The pressure of distillation was maintained at $17 \mathrm{hPa}$. Redistillation of the middle fraction repeated twice, gave pure ethyl butyl phosphite 1a (18.7 g, 18.8\%), or pure ethyl iso-butyl phosphite $\mathbf{1 b}$ (8.5 g, 8.5\%). The purity of compounds $\mathbf{1 a}$ and $\mathbf{1 b}$ was monitored by TLC (Thin Layer Chromatography) using ethyl acetate/hexane/isopropanol (4:7:1 v/v) as eluent.

1a:

${ }^{1} \mathbf{H}$ NMR $\left(\mathrm{CDCl}_{3}, \delta, \mathrm{ppm}\right): 0.93(\mathrm{t}, J=7.4 \mathrm{~Hz}, 3 \mathrm{H}$, $\mathrm{POCH}_{2} \mathrm{CH}_{2} \mathrm{CH}_{2} \mathrm{C}_{3}$ ), 1.37 (t, $J=7.1 \mathrm{~Hz}, 3 \mathrm{H}, \mathrm{POCH}_{2}$ $\left.\mathrm{C} \underline{H}_{3}\right), 1.38-1.48\left(\mathrm{~m}, 2 \mathrm{H}, \mathrm{POCH}_{2} \mathrm{CH}_{2} \mathrm{C}_{2} \mathrm{CH}_{3}\right), 1.64-1.73$ (m, $2 \mathrm{H}, \mathrm{POCH}_{2} \mathrm{C}_{2} \mathrm{CH}_{2} \mathrm{CH}_{3}$ ), 4.05-4.20 (m, $4 \mathrm{H}, \mathrm{POC} \underline{H}_{2}$ $\left.\mathrm{CH}_{2} \mathrm{CH}_{2} \mathrm{CH}_{3}, \mathrm{POC} \underline{H}_{2} \mathrm{CH}_{3}\right), 6,81(\mathrm{~d}, J=692.4 \mathrm{~Hz}, 1 \mathrm{H}$, $\mathrm{P} \underline{H}$ ).

${ }^{31} \mathbf{P}$ NMR $\left(\mathrm{CDCl}_{3}, \delta, \mathrm{ppm}\right): 8.23$. 1b:

${ }^{1} \mathbf{H}$ NMR $\left(\mathrm{CDCl}_{3}, \delta, \mathrm{ppm}\right): 0.96(\mathrm{~d}, J=6.7 \mathrm{~Hz}, 6 \mathrm{H}$, $\left.\mathrm{POCH}_{2} \mathrm{CH}\left(\mathrm{C}_{3}\right)_{2}\right), 1.37$ (t, $J=7.1 \mathrm{~Hz}, 3 \mathrm{H}, \mathrm{POCH}_{2}$ $\left.\mathrm{C} \underline{H}_{3}\right), 1.87-2.06\left(\mathrm{~m}, 2 \mathrm{H}, \mathrm{POCH}_{2} \mathrm{C} \underline{H}\left(\mathrm{CH}_{3}\right)_{2}\right), 3.77-3.91$ (m, $\left.2 \mathrm{H}, \mathrm{POC} \underline{H}_{2} \mathrm{CH}\left(\mathrm{CH}_{3}\right)_{2}\right), 4.06-4.21\left(\mathrm{~m}, 2 \mathrm{H}, \mathrm{POC} \underline{H}_{2}\right.$ $\left.\mathrm{CH}_{3}\right), 6.82(\mathrm{~d}, J=695.5 \mathrm{~Hz}, 1 \mathrm{H}, \mathrm{PC} \underline{H})$.

${ }^{31} \mathbf{P}$ NMR $\left(\mathrm{CDCl}_{3}, \delta, \mathrm{ppm}\right): 8.61$.

\section{Synthesis of compounds 2}

Compounds $\mathbf{1 a}$ and $\mathbf{1 b}$ were converted to $\mathbf{2 a}$ and $\mathbf{2 b}$ according to a modified method (Caplan, 2000).

$3.32 \mathrm{~g}(20 \mathrm{mmol})$ of ethyl butyl phosphite (or ethyl iso-butyl phosphite) was mixed with $1.84 \mathrm{~g}(20 \mathrm{mmol})$ glyoxylic acid monohydrate and $2.79 \mathrm{ml}(20 \mathrm{mmol})$ triethylamine. The resulting solution was stirred for $2 \mathrm{~h}$ at RT. Thereafter, the reaction mixture was dissolved in $10 \mathrm{ml}$ of distilled water and triethylamine was removed from the solution by ion exchange column (Dowex ${ }^{\circledR}$ 50W X8 50-100 mesh). After purification, $2.93 \mathrm{~g}$ (61\% yield) of pure 2-butyryloxy-2-[butoxyethoxyphosphinyl]acetic acid $2 \mathrm{a}$, or $3.59 \mathrm{~g}$ (75\% yield) of pure 2-butyryloxy2-[iso butoxyethoxyphosphinyl]acetic acid $\mathbf{2 b}$ were obtained.

2a:

${ }^{1} \mathbf{H}$ NMR $\left(\mathrm{CDCl}_{3}, \delta, \mathrm{ppm}\right): 0.94(\mathrm{t}, J=7.4 \mathrm{~Hz}, 3 \mathrm{H}$, $\mathrm{POCH}_{2} \mathrm{CH}_{2} \mathrm{CH}_{2} \mathrm{C}_{3}$ ), 1.37 (t, $J=7.1 \mathrm{~Hz}, 3 \mathrm{H}, \mathrm{POCH}_{2}$ $\mathrm{C} \underline{H}_{3}$ one pair of enantiomers), $1.38(\mathrm{t}, J=7.1 \mathrm{~Hz}, 3 \mathrm{H}$, $\mathrm{POCH}_{2} \mathrm{C}_{3}$ the second pair of enantiomers), 1.35-1.49 (m, $\left.2 \mathrm{H}, \mathrm{POCH}_{2} \mathrm{CH}_{2} \mathrm{C}_{2} \mathrm{CH}_{3}\right), 1.64-1.75\left(\mathrm{~m}, 2 \mathrm{H}, \mathrm{POCH}_{2}\right.$ $\mathrm{C}_{2} \mathrm{CH}_{2} \mathrm{CH}_{3}$ ), 4.18-4.34 (m, $4 \mathrm{H}, \mathrm{POC} \underline{H}_{2} \mathrm{CH}_{2} \mathrm{CH}_{2} \mathrm{CH}_{3}$, $\mathrm{POC} \underline{H}_{2} \mathrm{CH}_{3}$ ), 4.57 (d, $J=16.1 \mathrm{~Hz}, 1 \mathrm{H}, \mathrm{PC} \underline{H}$ ).

${ }^{31} \mathbf{P}$ NMR ( $\mathrm{CDCl}_{3}, \delta$, ppm): 16.80 .

${ }^{13} \mathrm{C}$ NMR $\left(\mathrm{CDCl}_{3}, \delta, \mathrm{ppm}\right): 13.74\left(\mathrm{POCH}_{2} \mathrm{CH}_{2} \mathrm{CH}_{2}\right.$ $\left.\underline{C} \mathrm{H}_{3}\right), 16.51\left(\mathrm{~d}, J=6.2 \mathrm{~Hz}, \mathrm{POCH}_{2} \underline{C} \mathrm{H}_{3}\right), 18.78$

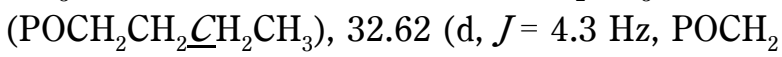
$\left.\underline{C} \mathrm{H}_{2} \mathrm{CH}_{2} \mathrm{CH}_{3}\right), 64.62\left(\mathrm{~d}, J=7.0 \mathrm{~Hz}, \mathrm{PO} \underline{C} \mathrm{H}_{2} \mathrm{CH}_{3}\right.$ one pair of enantiomers), 65.06 (d, $J=7.2 \mathrm{~Hz}, \mathrm{PO}_{\underline{C}} \mathrm{H}_{2}$ $\mathrm{CH}_{3}$ a second pair of enantiomers), 68.21 (d, $J=7.4 \mathrm{~Hz}, \mathrm{PO}_{\underline{C}} \mathrm{H}_{2} \mathrm{CH}_{2} \mathrm{CH}_{2} \mathrm{CH}_{3}$ one pair of enantiomers), 68.62 (d, $J=156.9 \mathrm{~Hz}, \mathrm{P} \underline{\mathrm{CH}}$ one pair of enantiomers), 68.65 (d, $J=7.5 \mathrm{~Hz}, \mathrm{PO} \underline{C} \mathrm{H}_{2} \mathrm{CH}_{2} \mathrm{CH}_{2} \mathrm{CH}_{3}$ a second pair of enantiomers), 68.66 (d, $J=$ $156.9 \mathrm{~Hz}, \mathrm{P} \underline{\mathrm{C}} \mathrm{H}$ a second pair of enantiomers), $170.55(\mathrm{~d}, J=10.3 \mathrm{~Hz}, \underline{\mathrm{COOH}})$.

2b:

${ }^{1} \mathbf{H}$ NMR $\left(\mathrm{CDCl}_{3}, \delta, \mathrm{ppm}\right): 0.96(\mathrm{~d}, J=6.6 \mathrm{~Hz}, 6 \mathrm{H}$, $\left.\mathrm{POCH}_{2} \mathrm{CH}\left(\mathrm{C}_{3}\right)_{2}\right), 1.37$ (t, $J=7.4 \mathrm{~Hz}, 3 \mathrm{H}, \mathrm{POCH}_{2}$ 
$\left.\mathrm{C} \underline{H}_{3}\right), 1.84-2.08\left(\mathrm{~m}, 2 \mathrm{H}, \mathrm{POCH}_{2} \mathrm{C} \underline{\mathrm{H}}\left(\mathrm{CH}_{3}\right)_{2}\right), 3.90-4.03$ (m, $\left.2 \mathrm{H}, \mathrm{POC} \underline{H}_{2} \mathrm{CH}\left(\mathrm{CH}_{3}\right)_{2}\right), 4.22-4.36\left(\mathrm{~m}, 2 \mathrm{H}, \mathrm{POC} \underline{H}_{2}\right.$ $\left.\mathrm{CH}_{3}\right), 4.58(\mathrm{~d}, J=16.0 \mathrm{~Hz}, 1 \mathrm{H}, \mathrm{PC} \underline{\mathrm{H}})$.

${ }^{31} \mathbf{P}$ NMR ( $\left.\mathrm{CDCl}_{3}, \delta, \mathrm{ppm}\right): 17.10$ (one pair of enantiomers), 17.12 (a second pair of enantiomers).

${ }^{13} \mathrm{C}$ NMR $\left(\mathrm{CDCl}_{3}, \delta, \mathrm{ppm}\right): 16.17(\mathrm{~d}, J=4.9 \mathrm{~Hz}$, $\left.\mathrm{POCH}_{2} \underline{\underline{C}} \mathrm{H}_{3}\right), 18.67\left(2 \mathrm{C}, \mathrm{POCH}_{2} \mathrm{CH}\left(\mathrm{C}_{3}\right)_{2}\right), 29.31$ (d, $\left.J=6.0 \mathrm{~Hz}, \mathrm{POCH}_{2} \underline{\mathrm{C}} \mathrm{H}\left(\mathrm{C}_{3}\right)_{2}\right), 64.42(\mathrm{~d}, J=6.8 \mathrm{~Hz}$, $\mathrm{PO} \underline{\mathrm{C}} \mathrm{H}_{2} \mathrm{CH}_{3}$ one pair of enantiomers), 64.62 (d, $J=$ $6.8 \mathrm{~Hz}, \mathrm{PO} \underline{\mathrm{C}} \mathrm{H}_{2} \mathrm{CH}_{3}$ a second pair of enantiomers), $68.83(\mathrm{~d}, J=161.2 \mathrm{~Hz}, \mathrm{P} \underline{\mathrm{C}} \mathrm{H}$ one pair of enantiomers), 67.99 (d, $J=161.2 \mathrm{~Hz}, \mathrm{P} \underline{\mathrm{CH}}$ a second pair of enantiomers), 74.11 (d, $J=7.7 \mathrm{~Hz}, \mathrm{PO} \underline{\mathrm{C}} \mathrm{H}_{2} \mathrm{CH}\left(\mathrm{C} \underline{H}_{3}\right)_{2}$ one pair of enantiomers), $74.41(\mathrm{~d}, J=7.7 \mathrm{~Hz}$, $\mathrm{PO} \underline{\mathrm{C}} \mathrm{H}_{2} \mathrm{CH}\left(\mathrm{C}_{3}\right)_{2}$ a second pair of enantiomers), $171.31(\mathrm{~d}, J=10.3 \mathrm{~Hz}, \underline{\mathrm{COOH}})$.

\section{Synthesis of compounds 3}

Compounds $\mathbf{1 a}$ and $\mathbf{1 b}$ were converted to $\mathbf{3 a}$ and $\mathbf{3 b}$ without the isolation of hydroxyphosphonates $\mathbf{2 a}$ and $\mathbf{2 b}$ according to a modified method described in the literature (Caplan, 2000; Majewska, 2006).

$3.32 \mathrm{~g}(20 \mathrm{mmol})$ of ethyl butyl phosphite (or ethyl iso-butyl phosphite) was mixed with $1.84 \mathrm{~g}$ (20 mmol) glyoxylic acid monohydrate and $2.79 \mathrm{ml}(20 \mathrm{mmol})$ triethylamine. The resulting solution was stirred for $2 \mathrm{~h}$ at RT. Thereafter, the reaction mixture was dissolved in $100 \mathrm{ml}$ of chloroform, placed in an ice bath and $2.07 \mathrm{ml}$ (20 mmol) of butyryl chloride was slowly added dropwise. After completion of the reaction, which lasts for two days as monitored by TLC, the resulting solution was extracted with $100 \mathrm{ml}$ of distilled water, the organic phase was evaporated and the product was purified by column chromatography using dichloromethane:isopropanol in a ratio of 100:5 as eluent. After purification, $3.46 \mathrm{~g}$ (59\% yield) of pure 2-butyryloxy-2-[butoxyethoxyphosphinyl]acetic acid $\mathbf{2 a}$, or $3,94 \mathrm{~g}$ (67\% yield) of pure 2-butyryloxy-2-[isobutoxyethoxyphosphinyl] acetic acid $\mathbf{2 b}$ were obtained.

3a:

${ }^{1} \mathrm{H}$ NMR $\left(\mathrm{CDCl}_{3}, \delta, \mathrm{ppm}\right): 0.93(\mathrm{t}, J=7.4 \mathrm{~Hz}, 3 \mathrm{H}$, $\mathrm{POCH}_{2} \mathrm{CH}_{2} \mathrm{CH}_{2} \underline{\mathrm{C}}_{3}$ ), 0.98 (t, $J=7.4 \mathrm{~Hz}, 3 \mathrm{H}, \mathrm{CH}_{2} \mathrm{CH}_{2}$ $\left.\mathrm{C} \underline{H}_{3}\right), 1.36$ (t, $\left.J=7.0 \mathrm{~Hz}, 3 \mathrm{H}, \mathrm{POCH}_{2} \mathrm{C}_{3}\right), 1.61-1.77$ (m, $4 \mathrm{H}, \mathrm{CH}_{2} \mathrm{C}_{2} \mathrm{CH}_{3}, \mathrm{POCH}_{2} \underline{\mathrm{C}}_{2} \mathrm{CH}_{2} \mathrm{CH}_{3}$ ), 2.37-2.54 (m, $2 \mathrm{H}, \underline{\mathrm{C}}_{2} \mathrm{CH}_{2} \mathrm{CH}_{3}$ ), 4.13-4.40 (m, 4H, $\mathrm{POC} \underline{H}_{2} \mathrm{CH}_{2}$ $\left.\mathrm{CH}_{2} \mathrm{CH}_{3}, \mathrm{POC} \underline{H}_{2} \mathrm{CH}_{3}\right), 5.54(\mathrm{~d}, J=17.8 \mathrm{~Hz}, 1 \mathrm{H}, \mathrm{PC} \underline{\mathrm{H}})$.
${ }^{31} \mathbf{P}$ NMR ( $\left.\mathrm{CDCl}_{3}, \delta, \mathrm{ppm}\right): 14.77$.

${ }^{13} \mathrm{C}$ NMR ( $\left.\mathrm{CDCl}_{3}, \delta, \mathrm{ppm}\right): 13.53\left(2 \mathrm{C}, \mathrm{CH}_{2} \mathrm{CH}_{2} \underline{\mathrm{C}} \mathrm{H}_{3}\right.$, $\left.\mathrm{POCH}_{2} \mathrm{CH}_{2} \mathrm{CH}_{2} \underline{C} \mathrm{H}_{3}\right), 16.28$ (d, $J=5,9 \mathrm{~Hz}, \mathrm{POCH}_{2}$ $\left.\underline{C} \mathrm{H}_{3}\right), 18.30\left(\mathrm{CH}_{2} \underline{\mathrm{C}} \mathrm{H}_{2} \mathrm{CH}_{3}\right), 18.59\left(\mathrm{POCH}_{2} \mathrm{CH}_{2} \underline{C} \mathrm{H}_{2}\right.$ $\left.\mathrm{CH}_{3}\right), 32.35\left(\mathrm{~d}, J=6.1 \mathrm{~Hz}, \mathrm{POCH}_{2} \mathrm{CH}_{2} \mathrm{CH}_{2} \mathrm{CH}_{3}\right.$ ), $35.53\left(\underline{C} \mathrm{H}_{2} \mathrm{CH}_{2} \mathrm{CH}_{3}\right), 64.56\left(\mathrm{~d}, J=7.4 \mathrm{~Hz}, \mathrm{PO}^{\mathrm{C}} \mathrm{H}_{2}\right.$ $\mathrm{CH}_{3}$ one pair of enantiomers), $64.66(\mathrm{~d}, J=7.4 \mathrm{~Hz}$, $\mathrm{PO} \underline{\mathrm{C}} \mathrm{H}_{2} \mathrm{CH}_{3}$ a second pair of enantiomers), 67.92 (d, $J=161.2 \mathrm{~Hz}, \mathrm{P} \underline{\mathrm{C}} \mathrm{H}), 68.04\left(\mathrm{~d}, J=7.6 \mathrm{~Hz}, \mathrm{PO} \underline{C} \mathrm{H}_{2}\right.$ $\mathrm{CH}_{2} \mathrm{CH}_{2} \mathrm{CH}_{3}$ one pair of enantiomers), 68.14 (d, $J=7.6 \mathrm{~Hz}, \mathrm{PO} \underline{C} \mathrm{H}_{2} \mathrm{CH}_{2} \mathrm{CH}_{2} \mathrm{CH}_{3}$ a second pair of enantiomers), $166.45\left(\underline{\mathrm{COCH}_{2}} \mathrm{CH}_{2} \mathrm{CH}_{3}\right), 172.07$ (d, $J=10.3 \mathrm{~Hz}, \underline{C O O H})$.

3b:

${ }^{1} \mathbf{H}$ NMR $\left(\mathrm{CDCl}_{3}, \delta, \mathrm{ppm}\right): 0.94(\mathrm{~d}, J=6.5 \mathrm{~Hz}, 6 \mathrm{H}$, $\left.\mathrm{POCH}_{2} \mathrm{CH}\left(\mathrm{C}_{3}\right)_{2}\right), 0.98$ (t, $J=7,5 \mathrm{~Hz}, 3 \mathrm{H}, \mathrm{CH}_{2} \mathrm{CH}_{2}$ $\left.\mathrm{C} \underline{H}_{3}\right), 1.36\left(\mathrm{t}, J=7.1 \mathrm{~Hz}, 3 \mathrm{H}, \mathrm{POCH}_{2} \mathrm{C}_{3}\right), 1.64-1.77$ (m, $2 \mathrm{H}, \mathrm{CH}_{2} \mathrm{C}_{2} \mathrm{CH}_{3}$ ), 1.90-2.03 (m, $1 \mathrm{H}, \mathrm{POCH}_{2}$ $\left.\mathrm{C} \underline{H}\left(\mathrm{CH}_{3}\right)_{2}\right), 2.37-2.54\left(\mathrm{~m}, 2 \mathrm{H}, \mathrm{C}_{2} \mathrm{CH}_{2} \mathrm{CH}_{3}\right), 3.92-$ $3.99\left(\mathrm{~m}, 2 \mathrm{H}, \mathrm{POC} \underline{H}_{2} \mathrm{CH}\left(\mathrm{CH}_{3}\right)_{2}\right), 4.24-4.31(\mathrm{~m}, 2 \mathrm{H}$, POC $\underline{H}_{2} \mathrm{CH}_{3}$ ), 5.55 (d, $J=17,7 \mathrm{~Hz}, 1 \mathrm{H}, \mathrm{PC} \underline{H}$ ).

${ }^{31} \mathbf{P}$ NMR $\left(\mathrm{CDCl}_{3}, \delta, \mathrm{ppm}\right): 14.67$ (one pair of enantiomers), 14.68 (a second pair of enantiomers).

${ }^{13} \mathrm{C}$ NMR $\left(\mathrm{CDCl}_{3}, \delta, \mathrm{ppm}\right): 13.68\left(\mathrm{CH}_{2} \mathrm{CH}_{2} \underline{\mathrm{C}} \mathrm{H}_{3}\right)$, $16.45\left(\mathrm{~d}, J=6.0 \mathrm{~Hz}, \mathrm{POCH}_{2} \underline{\underline{C}} \mathrm{H}_{3}\right), 18.41\left(\mathrm{CH}_{2} \underline{\underline{C}} \mathrm{H}_{2}\right.$ $\left.\mathrm{CH}_{3}\right), 18.66\left(2 \mathrm{C}, \mathrm{POCH}_{2} \mathrm{CH}\left(\underline{\mathrm{C}} \mathrm{H}_{3}\right)_{2}\right), 29.28$ (d, $\left.J=6.0 \mathrm{~Hz}, \mathrm{POCH}_{2} \underline{\mathrm{CH}}\left(\mathrm{CH}_{3}\right)_{2}\right), 35.66\left(\underline{\mathrm{C}} \mathrm{H}_{2} \mathrm{CH}_{2} \mathrm{CH}_{3}\right)$, $64.83\left(\mathrm{~d}, J=5.6 \mathrm{~Hz}, \mathrm{PO} \underline{C} \mathrm{H}_{2} \mathrm{CH}_{3}\right.$ one pair of enantiomers), $64.90\left(\mathrm{~d}, J=5.6 \mathrm{~Hz}, \mathrm{PO} \underline{\mathrm{C}} \mathrm{H}_{2} \mathrm{CH}_{3}\right.$ a second pair of enantiomers), 67.95 (d, $J=161.2 \mathrm{~Hz}, \mathrm{P} \underline{\mathrm{C}} \mathrm{H}$ one pair of enantiomers), 67.99 (d, $J=161.2 \mathrm{~Hz}$, $\mathrm{P} \underline{\mathrm{CH}}$ a second pair of enantiomers), 74.10 (d, $J=6.2 \mathrm{~Hz}, \mathrm{PO} \underline{\mathrm{C}} \mathrm{H}_{2} \mathrm{CH}\left(\mathrm{CH}_{3}\right)_{2}$ one pair of enantiomers), $74.18\left(\mathrm{~d}, J=6.2 \mathrm{~Hz}, \mathrm{PO} \underline{C} \mathrm{H}_{2} \mathrm{CH}\left(\mathrm{CH}_{3}\right)_{2}\right.$ a second pair of enantiomers), $166.49\left(\underline{\mathrm{COCH}_{2}} \mathrm{CH}_{2} \mathrm{CH}_{3}\right)$, $172.17(\mathrm{~d}, J=10.3 \mathrm{~Hz}, \underline{C} \mathrm{OOH})$.

\section{Microorganisms, growth and whole cell biotransformation conditions}

Microorganisms were cultivated in a medium tested previously for stimulating vigorous growth and the lipolytic activity (Majewska et al., 2006).

One liter of the medium contained $10 \mathrm{~g}$ soluble starch, $1 \mathrm{~g}$ yeast extract, $5 \mathrm{~g}\left(\mathrm{NH}_{4}\right)_{2} \mathrm{SO}_{4}, 2 \mathrm{~g} \mathrm{~K}{ }_{2} \mathrm{HPO}$, and $100 \mu \mathrm{l}$ of tributyrin and 11 mixed in distilled water. The microorganisms were incubated at $26^{\circ} \mathrm{C}$ with shaking at $150 \mathrm{rpm}$ 


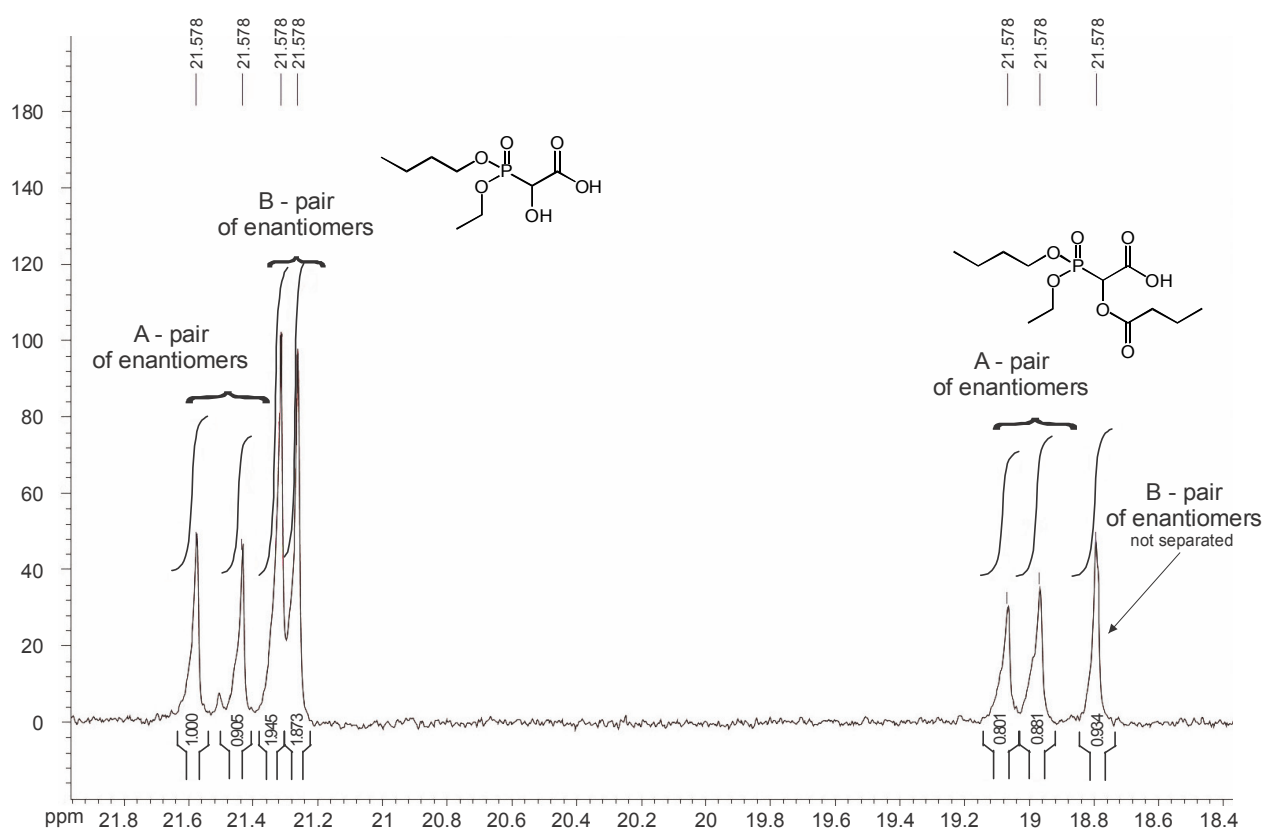

Fig. 3. ${ }^{31} \mathrm{P}$ NMR spectra with quinine as a chiral solvating agent of biotransformation reaction of 3a with Bacillus subtilis after 6 days. On the left, there is product - hydroxyphosphonate $\mathbf{2 a}$, on the right - unreacted substrate $\mathbf{3 a}$

for $24 \mathrm{~h}$. Subsequently, the cells were centrifuged at $1449 \times g$ for $10 \mathrm{~min}$. Biotransformations were performed in $100 \mathrm{ml}$ solution of $0.017 \mathrm{M}$ phosphate buffer, $\mathrm{pH} 7.0$, and $50 \mu \mathrm{l}$ of substrate with (150 rpm shaking; $\mathrm{RT}$ ). Then the biomass was centrifuged at $1449 \times g$, the supernatant evaporated and the products of biotransformation were extracted with acetonitrile three times. Thereafter, the organic solvent was evaporated and the products were analyzed by means of ${ }^{31} \mathrm{P}$ NMR spectroscopy using quinine as a chiral discriminator.

\section{Results and discussion}

Firstly it must be noted that the chemical shift of each isomer on ${ }^{31} \mathrm{P}$ NMR spectra is only tentative at this stage of research and the correlation of the signals on ${ }^{31} \mathrm{P}$ NMR spectra (with chiral solvating agent - quinine) and absolute configuration of isomers is unknown (Fig. 3). However, previous experiments on structurally similar compounds with the use of analogical method (Majewska et al., 2006; Majewska et al., 2009) and deductive analysis of the obtained data allowed us to cautiously assign NMR signals to eantiomers and diastereomers. The starting substrate is a mixture of four stereoisomers - two enantiomeric mixtures of diastereomers (denoted as A and B). Therefore, the conversion of each pair of enantiomers was analyzed separately.
In the case of compounds with one center of chirality, the process of ester hydrolysis should be terminated at conversion equal to $50 \%$ when the enzyme works with $100 \%$ enantioselectivity. In such a case, only one of the enantiomers is converted to hydroxyphosphonate. In the case of four stereoisomers however, this simple picture becomes far more complex. The reaction of hydrolysis can proceed enantioselectively or diastereoselectively, where both selectivities can be observed at the same time.

Stereochemistry of compounds $\mathbf{2 a}, \mathbf{2 b}, \mathbf{3} \mathbf{a}$ and $\mathbf{3 b}$ is unknown and there are no data in the literature about it. Attempts to separate enantiomeric mixtures A and B by non-chiral methods such as chromatography proved unsuccessful. Some enantioselectivity was observed when $S$. liquefaciens was used as a biocatalyst, but it did not exceed 25\% (ee - enantiomeric excess, see Table 3). In other cases, only diastereoselectivity was observed (de - diastereomeric excess, see Table 1 and Table 2). B. subtilis appeared to carry out hydrolysis most effectively (see Table 1 and Fig. 2) compared with two other organisms (see Table 2 and Table 3 ) with conversion up to $80 \%$ after 6 days for compound 3a. B. subtilis hydrolyzed both compounds without enantioselectivity but with diastereo selectivity reaching $60 \%$. In addition, B. subtilis hydrolyzed the enantiomeric mixture denoted as A (one mixture of two enantiomers) less efficiently than 
Table 1. Biotransformations with Bacillus subtilis

\begin{tabular}{|c|c|c|c|c|c|}
\hline \multirow{2}{*}{ Compound } & \multirow{2}{*}{$\begin{array}{l}\text { Time } \\
\text { [days] }\end{array}$} & \multicolumn{2}{|c|}{ Conversion [\%] } & \multirow{2}{*}{$\begin{array}{c}\text { de of product } \\
{[\%]}\end{array}$} & \multirow{2}{*}{$\begin{array}{c}\text { de of substrate } \\
{[\%]}\end{array}$} \\
\hline & & A & B & & \\
\hline & 3 & 27 & 48 & 36 & 7 \\
\hline & 5 & 47 & 78 & 52 & 11 \\
\hline $3 a$ & 6 & 53 & 80 & 33 & 29 \\
\hline & 5 & 16 & 30 & 33 & 5 \\
\hline & 6 & 19 & 56 & 57 & 18 \\
\hline $3 b$ & 7 & 24 & 69 & 59 & 27 \\
\hline
\end{tabular}

Table 2. Biotransformations with Escherichia coli

\begin{tabular}{|c|c|c|c|c|c|}
\hline \multirow{2}{*}{ Compound } & \multirow{2}{*}{$\begin{array}{l}\text { Time } \\
\text { [days] }\end{array}$} & \multicolumn{2}{|c|}{ Conversion [\%] } & \multirow{2}{*}{$\begin{array}{c}\text { de of product } \\
{[\%]}\end{array}$} & \multirow{2}{*}{$\begin{array}{c}\text { de of substrate } \\
{[\%]}\end{array}$} \\
\hline & & A & B & & \\
\hline & 4 & 19 & 20 & - & - \\
\hline & 5 & 26 & 28 & - & - \\
\hline & 6 & 27 & 23 & 12 & - \\
\hline $3 a$ & 7 & 30 & 26 & 10 & - \\
\hline & 4 & 19 & 14 & 18 & - \\
\hline $3 b$ & 5 & 22 & 16 & 20 & - \\
\hline
\end{tabular}

Table 3. Biotransfomations with Serratia liquefaciens

\begin{tabular}{|c|c|c|c|c|c|c|c|}
\hline \multirow{2}{*}{ Compound } & \multirow{2}{*}{$\begin{array}{l}\text { Time } \\
\text { [days] }\end{array}$} & \multicolumn{2}{|c|}{ Conversion [\%] } & \multicolumn{2}{|c|}{ ee of product [\%] } & \multirow{2}{*}{$\begin{array}{c}\text { de of product } \\
{[\%]}\end{array}$} & \multirow{2}{*}{$\begin{array}{c}\text { de of substrate } \\
{[\%]}\end{array}$} \\
\hline & & A & B & A & B & & \\
\hline & 4 & 20 & 30 & 10 & - & 25 & - \\
\hline & 5 & 20 & 28 & 19 & 10 & 26 & - \\
\hline & 6 & 11 & 25 & 25 & - & 46 & - \\
\hline $3 a$ & 7 & 13 & 31 & 22 & - & 48 & - \\
\hline & 4 & 14 & 24 & 14 & 9 & 30 & - \\
\hline $3 b$ & 7 & 16 & 26 & 10 & 10 & 27 & - \\
\hline
\end{tabular}


the mixture denoted as $\mathrm{B}$ (the second mixture of two enantiomers) for both compounds: $\mathbf{3 a}$ and $\mathbf{3 b}$. The highest dewas obtained for compound $\mathbf{3 a}$ after five days of reaction $(52 \%)$ with reaction yield reaching $47 \%$. When the yield of reaction exceeded $50 \%$, stereospecificity dropped significantly (to $33 \%$ ). In the case of compound $\mathbf{3 b}$, better stereospecificities were obtained, albeit with lower yields of reaction.

\section{Conclusions}

Among the three studied microorganisms, only $B$. subtilis appeared to be a moderate biocatalyst in terms of stereopreference of hydroxyphosphonate production. The interesting finding of this work is that this biocatalyst hydrolyzed isomers of tested compounds with both ( $R$ and $S$ ) configurations located at carbon atom. Usually, this kind of reaction is more or less enantioselective rather than diastereoselective.

\section{Acknowledgements}

This work was financed from the Project "Biotransformations for pharmaceutical and cosmetics industry" No. POIG.01.03. 01-00-158/09-07 partly-financed by the European Union within the European Regional Development Fund for Innovative Economy.

\section{References}

Caplan N.A., Pogson Ch.I., Hayes D.J., Blackburn G.M. (2000) The synthesis of novel bisphosphonates as inhibitors of phosphoglycerate kinase (3-PGK). J. Chem. Soc., Perkin Trans. 1: 421-437.

Kategaonkar A.H., Pokalwar R.U., Sonar S.S., Gawali V.U. (2010) Synthesis, in vitro antibacterial and antifungal evaluations of new $\alpha$-hydroxyphosphonate and new $\alpha$-acetoxyphosphonate derivatives of tetrazolo [1, 5-a] quinoline. Eur. J. Med. Chem. 45: 1128-1132.

Klimek-Ochab M., Żymańczyk-Duda E., Brzezińska-Rodak M., Majewska P., Lejczak B. (2008) Effective fungal catalyzed synthesis of P-chiral organophosphorus compounds. Tetrahedron: Asymm. 19: 450-453.

Kolodiazhnyi O.I. (2005) Asymmetric synthesis of hydroxyphosphonates. Tetrahedron: Asymm. 16: 3295-3340.
Kolodiazhnyi O.I. (2012) Recent developments in the asymmetric synthesis of P-chiral phosphorus compounds. Tetrahedron: Asymm. 23: 1-46.

Kosolapoff G.M. (1951) Preparation of Some Mixed Dialkyl Phosphites. J. Am. Chem. Soc. 73: 4989.

Magee W.C., Evans D.H. (2012) The antiviral activity and mechanism of action of (S)-[3-hydroxy-2-(phosphonomethoxy) propyl] (HPMP) nucleosides. Antivir. Res. 96: 169-180.

Majewska P., Kafarski P., Lejczak B., Bryndal I., Lis T. (2006) An approach to the synthesis and assignment of the absolute configuration of all enantiomers of ethyl hydroxy(phenyl)methane(P-phenyl)phosphinate. Tetrahedron: Asymm. 17: 2697-2701.

Majewska P., Kafarski P., Lejczak B. (2006) Simple and effective method for the deracemization of ethyl 1-hydroxyphosphinate using biocatalysts with lipolytic activity. Tetrahedron: Asymm. 17: 2870-2875.

Majewska P., Doskocz M., Lejczak B., Kafarski P. (2009) Enzymatic resolution of a-hydroxyphosphinates with two stereogenic centers and determination of absolute configuration of obtained stereoisomers. Tetrahedron: Asymm. 20: $1568-1574$.

Malinowska B., Majewska P., Szatkowski P., Kafarski P., Lejczak B. (2011) Kinetic resolution of ( \pm )-diethyl- and dibenzyl hydroxy(phenyl) methanephosphonates and their acyl derivatives with lipases. Biocatal. Biotrans. 29: 271277.

Nesterov V.V., Kolodiazhnyi O.I. (2006) New method for the asymmetric hydroboration of ketophosphonates and the synthesis of phospho-carnitine. Tetrahedron: Asymm. 17: 1023-1026.

Pawar V.D., Bettigeri S., Weng S.-S., Kao J.-Q., Chen C.-T. (2006) Highly Enantioselective Aerobic Oxidation of $r$ Hydroxyphosphonates Catalyzed by Chiral Vanadyl(V) Methoxides Bearing N-Salicylidene-r-aminocarboxylates. J. Am. Chem. Soc. 128: 6308-6309.

Plażuk D., Zakrzewski J., Rybarczyk-Pirek A. (2006) Resolution and absolute configuration of dimethyl hydroxy(ferrocenylmethyl)phosphonate. Tetrahedron: Asymm. 17: 1975-1978.

Skwarczyński M., Lejczak B., Kafarski P. (1999) Enantioselective Hydrolysis of 1-Butyryloxyalkylphosphonates by Lipolytic Microorganisms: Pseudomonas fluorescens and Penicillium citrinum. Chirality 11: 109-114. 